T. Sasaki and K. Shiga

Nagoya Math. J.

Vol. 71 (1978), 91-95

\title{
REMARKS ON THE CURVATURE OF HYPERSURFACES IN $\boldsymbol{C}^{n}$
}

\author{
TAKESHI SASAKI AND KIYOSHI SHIGA
}

\section{§1. Introduction}

In this paper we shall study the global condition of curvatures of hypersurfaces in the complex Euclidean space. S. Bochner ([1]) and E. Calabi ([2]) have already investigated kähler embeddings (i.e. holomorphic and isometric embeddings) of a kähler manifold into the complex Euclidean space. However, it seems that they did not discuss the relation between the embeddability and the curvature.

It is well known that both the complex line and the unit disc can be embedded holomorphically in the two dimensional complex Euclidean space as a closed complex submanifold. While there is no complete kähler structure on the complex line, whose curvature function is bounded by a negative constant from above. Therefore the complex line cannot be immersed in $C^{2}$ so that the curvature of the induced metric, if complete, would be bounded from above by a negative constant. As for the unit disc we shall encounter the same situation (Theorem 1). The proof will be given by considering the Gauss map associated with the immersion and this argument can be also applied to the embedding problem of hypersurfaces in $\boldsymbol{C}^{n}$.

\section{$\S 2$. Curves in the complex Euclidean space}

Let $C^{n}$ be the $n$-dimensional complex Euclidean space and $\iota: C \rightarrow C^{n}$ be an immersion of a complex curve $C$. First we shall define the Gauss map. The tangent space of $C^{n}$ at $p: T_{p}\left(C^{n}\right)$ can be canonically identified with $\boldsymbol{C}^{n}$ through the differential $\iota_{*}$ of $\iota$. Denote by $\boldsymbol{P}^{n-1}$ the complex projective space, i.e. the set of all complex lines in $C^{n}$. Then we can define the map

$$
\phi: C \rightarrow \boldsymbol{P}^{n-1}
$$

Received June 20, 1977. 
by $\phi(p)=\iota_{*} T_{p}(C) \in P^{n-1}$ for $p \in C$, which is called the Gauss map associated with the immersion $\iota$.

Let $d \tilde{s}^{2}$ (resp. $d s^{2}$ ) be the Fubini-Study metric on $\boldsymbol{P}^{n-1}$ (resp. the metric on $C$ induced by $\iota$ ). These two metrics have a remarkable relation with the Gauss map $\phi$, which we shall state in the following. Fix a point $p \in C$. In the neighbourhood $U$ of $\iota(p)$ we may take $z_{1}$ as the local coordinate of $\iota(C)$ when $\left(z_{1}, \cdots, z_{n}\right)$ is the linear coordinate of $C^{n}$ (make an appropriate unitary transformation, if necessary, on $C^{n}$ ). Hence there exist holomorphic functions $f_{2}, \ldots, f_{n}$ such that $c(C) \cap U$ $=\left\{z \in U \mid z_{2}=f_{2}\left(z_{1}\right), \cdots, z_{n}=f_{n}\left(z_{1}\right)\right\} . \quad$ Then $T_{p}(C) \equiv C\left\{\frac{\partial}{\partial z_{1}}+\sum_{i=2}^{n} \frac{\partial f_{i}}{\partial z_{1}} \frac{\partial}{\partial z_{i}}\right\}$. This means that, if we denote by $\left[z_{1}, \cdots, z_{n}\right]$ the homogeneous coordinate of $\boldsymbol{P}^{n-1}$,

$$
\phi\left(z_{1}\right)=\left[1, \frac{\partial f_{2}}{\partial z_{1}}, \cdots, \frac{\partial f_{n}}{\partial z_{1}}\right]
$$

Therefore $d s^{2}=h d z_{1} \overline{d z_{1}}$, where $h=1+\sum_{j=2}^{n} \frac{\partial f_{j}}{\partial z_{1}} \frac{\overline{\partial f_{j}}}{\partial z_{1}}$. The Gauss curvature $\kappa$ of this metric is given by

$$
\kappa=-\frac{1}{h} \frac{\partial^{2}}{\partial z_{1} \partial \bar{z}_{1}} \log h .
$$

On the other hand the Fubini-Study metric is given by

$$
d \tilde{s}^{2}=4 \frac{\partial^{2}}{\partial z_{i} \partial \bar{z}_{k}} \log \left(1+\sum_{j=2}^{n} z_{j} \bar{z}_{j}\right) d z_{i} \overline{d z_{k}}
$$

on the open set $\left\{z_{1} \neq 0\right\}$ in $P^{n-1}$ (cf. [3], p. 160). Hence by (1) we get

$$
\begin{aligned}
\phi^{*} d \tilde{s}^{2} & =4 \frac{\partial^{2}}{\partial z_{1} \partial \bar{z}_{1}} \log \left(1+\sum_{j=2}^{n} \frac{\partial f_{j}}{\partial z_{1}} \overline{\frac{\partial f_{j}}{\partial z_{1}}}\right) d z_{1} \overline{d z_{1}} \\
& =4 \frac{1}{h}\left(\frac{\partial^{2}}{\partial z_{1} \partial \bar{z}_{1}} \log h\right) h d z \overline{d z_{1}} \\
& =-4 \kappa d s^{2} .
\end{aligned}
$$

This is the so-called Rodrigues' formula.

In this situation we shall prove

THEOREM 1. Let $C$ be a complete kähler manifold of dimension 1. Assume there exists a negative constant $-k(k>0)$ such that the Gauss 
curvature is less than $-k$. Then $C$ has no kähler immersion into $C^{2}$.

Proof. Assume $C$ has a kähler immersion into $C^{2}$. For the associated Gauss map $\phi$ the relation (4) holds. Since $d s^{2}$ is complete and $\kappa<-k<0, \phi^{*} d \tilde{s}^{2}$ is a complete kähler metric on $C$. It is obviously of constant positive curvature and $\phi$ is a local diffeomorphism. Therefore $C$ must be compact, which is a contradiction, and hence the theorem is proved.

\section{§3. Hypersurfaces in the complex Euclidean space}

We shall define the Gauss map of a hypersurface in $C^{n+1}$. Let $\iota: S \rightarrow C^{n+1}$ be an immersion of a complex manifold $S$ of dimension $n$. If we take appropriately the linear coordinate $\left(z_{1}, \cdots, z_{n+1}\right)$ of $C^{n+1}, S$ can be locally identified with the hypersurface

$$
\left\{z \in C^{n+1} \mid z_{n+1}=f\left(z_{1}, \cdots, z_{n}\right)\right\}
$$

for some holomorphic function $f$ of $\left(z_{1}, \cdots, z_{n}\right)$. The induced metric $d s^{2}$ on $S$ is given by

$$
\begin{aligned}
d s^{2} & =d z_{1} \overline{d z_{1}}+\cdots+d z_{n} \overline{d z_{n}}+d f \overline{d f} \\
& =\sum_{i, j}\left(\delta_{i j}+f_{i} \overline{f_{j}}\right) d z_{i} \overline{d z_{j}}
\end{aligned}
$$

with respect to the local coordinate $\left(z_{1}, \cdots, z_{n}\right)$, where $f_{i}=\frac{\partial f}{\partial z_{i}}$. Let $G_{n, 1}$ be the Grassman manifold of all hyperplanes in $\boldsymbol{C}^{n+1}$. The elements of $\boldsymbol{G}_{n, 1}$ can be written as follows. To every $n \times(n+1)$-matrix $Z$ of rank $n$ there corresponds a hyperplane determined by $n$ column vectors of $Z$. Writing $Z=\left(\begin{array}{c}Z_{0} \\ T\end{array}\right)$, where $Z_{0}$ is a $n \times n$-matrix and $T$ is a row vector, we consider the open set $U$ of $G_{n, 1}$ defined by $\operatorname{det} Z_{0} \neq 0$. In this respect every element of $U$ is determined by one and only one matrix of the form $\left(\begin{array}{l}I \\ T\end{array}\right)$, where $I$ is the unit matrix, and $T$ gives a local coordinate of $U$. Then the Fubini-Study metric form $\Phi$ on $U$ is given by

$$
\Phi=-4 i \partial \bar{\partial} \log \operatorname{det}\left(I+{ }^{t} \bar{T} T\right),
$$

(cf. [3], p. 161).

Now for the hypersurface $S$ we define the Gauss map 


$$
\phi: S \rightarrow \boldsymbol{G}_{n, 1}
$$

by $\phi(p)=\iota_{*} T_{p}(S)$. This definition is essentially the same one defined by A. Vitter [4]. Since $T_{p}(S)$ is spanned by $n$-vectors

$$
\frac{\partial}{\partial z_{i}}+f_{i} \frac{\partial}{\partial z_{n+1}}, \quad i=1, \cdots, n,
$$

$\phi$ can be written as

$$
\phi\left(z_{1}, \cdots, z_{n}\right)=\left(f_{1}, \cdots, f_{n}\right)
$$

with respect to the above-mentioned coordinate of $G_{n, 1}$. On the other hand the Ricci form $\rho$ of the induced kähler metric $d s^{2}$ is

$$
\rho=-2 i \partial \bar{\partial} \log \operatorname{det}\left(\delta_{i \jmath}+f_{i} \overline{f_{j}}\right) .
$$

Therefore

$$
\phi^{*} \Phi=-2 \rho .
$$

THEOREM 2. Let $S$ be a complete kähler manifold of dimension $n$. If there exists a negative constant $-k(k>0)$ such that $\rho \leq-k \omega$, where $\omega$ (resp. $\rho$ ) is the kähler (resp. Ricci) form of $S$, then $S$ has no kähler immersion into $\boldsymbol{C}^{n+1}$.

Proof. Assume $S$ is immersed in $C^{n+1}$ and let $\phi: S \rightarrow G_{n, 1}$ be the associated Gauss map. Then by (10) and the assumption we have $\phi^{*} \Phi$ $>2 k \omega$. Hence $\phi$ is non-degenerate and $\phi^{*} \Phi$ defines a complete kähler metric on $S$. Since $\Phi$ is of constant holomorphic sectional curvature, $S$ can be covered by a complex space form of positive curvature. Therefore $S$ must be compact, which is a contradiction.

By Theorem 2 we know that the $n$-dimensional complete kähler manifold cannot be immersed in $C^{n+1}$ if the holomorphic bisectional curvature is bounded by a negative constant from above, because every Ricci curvature is the sum of holomorphic bisectional curvatures. More generally we have

COROLlaRY. Let $S$ be a complete kähler manifold of dimension $n$. If there exists a negative constant $-k(k>0)$ such that every holomorphic sectional curvature of $S$ is less than $-k$, then $S$ has no kähler immersion into $\boldsymbol{C}^{n+1}$. 
Proof. Assume $S$ has a kähler immersion $\iota: S \rightarrow C^{n+1}$. If we take a generic hyperplane $H$, then $\iota: S \cap \iota^{-1}(H) \rightarrow H$ is also a kähler immersion. Continuing $(n-1)$-times this procedure we have a 2-dimensional linear subspace $L \subset C^{n+1}$ such that $\iota: S \cap c^{-1}(L) \rightarrow L$ is an immersion of a curve in $L$. Since the holomorphic sectional curvature of a submanifold does not exceed that of the ambient space, the Gauss curvature of the induced metric on $S \cap c^{-1}(L)$ is less than $-k$, which contradicts to Theorem 1.

Added in proof (August 7, 1978). After this paper was submitted to publication, the authors were informed of, kindly by $\mathrm{H}$. Wu, the analogous results of $\mathrm{P}$. Yang which have announced in Proc. of Symp. in pure Math. 30-II (1977), 135-137. Also see his paper in J. of Differential Geometry (to appear).

\section{REFERENCES}

[1] S. Bochner, Curvature in hermitian metric, Bull. Amer. Math. Soc., 53 (1947), 179-195.

[2] E. Galabi, Isometric imbedding of complex manifolds, Ann. of Math., 58 (1953), $1-23$.

[ 3 ] S. Kobayashi and K. Nomizu, Foundations of differential geometry, vol. II (1969), Interscience publishers.

[4] A. Vitter, On the curvature of complex hypersurfaces, Indiana Univ. Math. J., 23 (1974), 813-826.

Nagoya University

Gifu University 